\title{
Zirconium Zr 89 Pembrolizumab
}

National Cancer Institute

\section{Source}

National Cancer Institute. Zirconium Zr 89 Pembrolizumab. NCI Thesaurus. Code C148167.

A radioimmunoconjug ate composed of pembrolizumab, a humanized monoclonal antibody immunoglobulin (Ig) G4 antibody directed against the human cell surface receptor programmed cell death 1 (PD-1; PDCD1; CD279; programmed cell death-1), labeled with the radioisotope zirconium Zr 89, with potential use as an imaging agent upon positron emission tomography (PET). Upon administration of zirconium Zr 89 pembrolizumab, the antibody moiety binds to PD-1, an inhibitory signaling receptor expressed on the surface of activated T-cells, and blocks the binding to and activation of PD-1 by its ligands. Upon PET imaging, PD-1-expressing T-cells, as well as pembrolizumab biodistribution, can be visualized and the expected response to treatment with pembrolizumab, as well as selection of patients that would respond to pembrolizumab, can be assessed. The ligands for PD-1 include programmed cell death-1 lig and 1 (PD-L1), which is overexpressed on certain cancer cells, and prog rammed cell death-1 ligand 2 (PD-L2), which is primarily expressed on antigen-presenting cells (APCS). Activated PD-1 negatively regulates T-cell activation and plays a key role in tumor evasion from host immunity. 